\title{
Amylase action pattern on starch polymers
}

\author{
Annabel Bijttebier*, Hans Goesaert* \& Jan A. Delcour \\ Laboratory of Food Chemistry and Leuven Food Science and Nutrition Research Centre (LFoRCe), Kasteelpark Arenberg 20, \\ B-3001 Leuven, Belgium; e-mails: Annabel.Bijttebier@biw.kuleuven.be; hans.goesaert@biw.kuleuven.be
}

\begin{abstract}
Several decades ago, the first reports on differences in action pattern between amylases from different sources indicated that the starch polymers are not degraded in a completely random manner. We here give an overview of different action patterns of amylases on amylose and amylopectin, focusing on the so-called multiple attack action of the enzymes. Nowadays, the multiple attack action is generally an accepted concept to explain the differences in amylase action pattern. However, the pancreatic $\alpha$-amylase remains one of the few enzymes known with a considerable level of multiple attack action. Despite some recent studies, the molecular mechanism of the multiple attack action is still largely unclear. Probably, the degree to which the active site architecture and binding properties allow both the reorganization (sliding) of the substrate in the active site and the stabilisation of the productive enzyme/substrate complex mainly determine the multiple attack action of amylases.
\end{abstract}

Key words: amylase; amylose; amylopectin; action pattern; multiple attack action.

Abbreviations: BAA, Bacillus amyloliquefaciens $\alpha$-amylase; BLA, Bacillus licheniformis $\alpha$-amylase; BSuA, Bacillus subtilis $\alpha$-amylase; BStA, Bacillus stearothermophilus maltogenic $\alpha$-amylase; DMA, degree of multiple attack; DP, degree of polymerisation; GH, glycoside hydrolase; MW, molecular weight; PPA, porcine pancreatic $\alpha$-amylase; RV $\mathrm{RS}_{\mathrm{TS}}$, total level of reducing sugars; $\mathrm{RV}_{\mathrm{PS}}$, level of reducing polysaccharides; $\mathrm{SBD}$, starch binding domain; TAKA, Aspergillus oryzae $\alpha$-amylase; $\lambda_{\max }$, wavelength of maximal extinction.

\section{Introduction}

The amylase-catalysed hydrolysis of starch is among the most important industrially applied enzyme reactions (Gupta et al. 2003). The susceptibility of starch to amylase attack depends on the properties of the specific starch, such as e.g. degree of gelatinization, and the characteristics of the specific amylase. Many studies deal with the amylolysis of native starch and focus on the effects of substrate characteristics, such as granule size, shape and structure and amylose content. In addition, amylases showed widely different activities on various kinds of solubilised starches (Mukerjea et al. 2006). Amylases are, hence, often defined by their starch degrading characteristics and less by their action on the individual starch polymers. It is often accepted that amylases mainly randomly hydrolyse amylose and amylopectin. However, the finding that amylases from various sources degrade starch in a different, non-random manner has first been discussed several decades ago. We here give an overview of the different action patterns of amylases on amylose and amylopectin, focusing on the so-called multiple attack action of the enzymes.

\section{The substrate: amylose and amylopectin}

The native starch granule is mainly made up of two glucose polymers, i.e. amylose and amylopectin. Amylopectin is, in general, the major fraction of starch, with levels ranging from 75 to $85 \%$ (Manners 1979) for normal starch to even more than $99 \%$ for waxy varieties, which are essentially amylose free (Singh et al. 2003). High amylose mutant starches exist as well with amylose levels ranging from 40 to $80 \%$ (Manners 1979; Jane et al. 1999; Song \& Jane 2000).

Amylose is an essentially linear starch polymer of $\alpha$-(1-4) linked D-glucopyranosyl units with very few $\alpha$ (1-6) branch points (Hizukuri et al. 1981; Buléon et al. 1998). It has a degree of polymerisation (DP) in the range of 500-6000 glucose residues. Amylose can form inclusion complexes with e.g. lipids, alcohols and iodine (Zobel 1988). With iodine, a coloured complex is formed, with colour intensity and wavelength for maximal extinction $\left(\lambda_{\max }\right)$ depending on the concentration and average chain length of amylose (Bailey \& Whelan 1961; Banks et al. 1971). The minimum chain length necessary for iodine colour formation is 18 glucose units (Bailey \& Whelan 1961). Intensity and $\lambda_{\max }$ increase linearly with chain length to about 70 glucose units. Concomitantly, the colour changes from brown to red to purple and finally blue (at DP 45 and up).

Amylopectin is a branched starch polymer consisting of many short chains, each made up by $\alpha$-(1-4)-linked D-glucopyranosyl units, which are inter-

* Corresponding author 


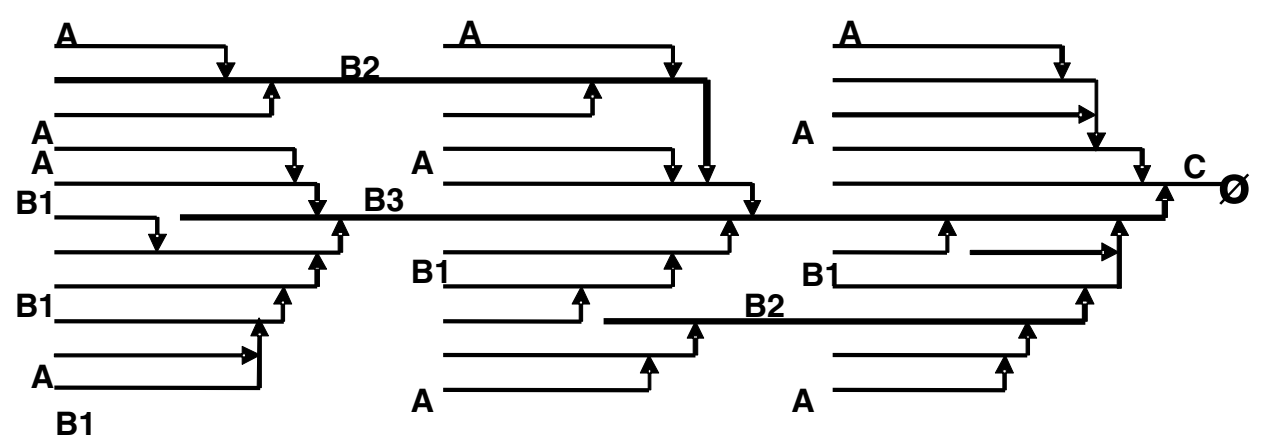

Fig. 1. Structure of amylopectin, identifying A, B and C chains. Adapted from Hizukuri $(1986,1996)$. Solid line: $\alpha-1,4$ bound glucose units; arrow: $\alpha-1,6$ linkage; $\varnothing$ : reducing glucose residue.

linked by $\alpha$-(1-6) bonds. It is one of the largest polymers in Nature, with a DP ranging from $3 \times 10^{5}$ to $3 \times 10^{6}$ glucose units (Zobel 1988). However, the individual amylopectin chains vary between 10 and 100 glucose units (Manners 1979). Nowadays, the cluster based amylopectin structure model is widely accepted (Fig. 1). In this model, the linear A-chains (DP 12-16) are linked to linear B-chains, which, in their turn, are linked to other B-chains or to the C-chain, which carries the only reducing glucose residue. The B-chains can be further divided into B1- (DP 20-24), B2- (DP 42-48), B3- (DP 69-75) and B4-chains, depending on the number of clusters they belong to (Hizukuri 1986, 1996).

\section{The enzyme: amylases}

\section{Classification}

Amylases are glycoside hydrolases (GHs), which act upon the bonds between the glucose units of the starch polymers. Several GH classification systems exist. One of the simplest classifications is based on substrate specificity. Each enzyme is characterized by an EC number based on the recommendations of the International Union of Biochemistry and Molecular Biology. GHs are characterized by the number EC 3.2.1.x, with the $\mathrm{x}$ representing the substrate specificity or in some cases the molecular mechanism or type of linkage (e.g. EC 3.2.1.1, $\alpha$-amylase; EC 3.2.1.2, $\beta$-amylase; EC 3.2.1.3, glucoamylase). Difficulties arise with this substrate specificity based classification, when an enzyme hydrolyses several substrates. Moreover, this classification does not reflect structural or mechanistic characteristics of enzymes (Henrissat \& Davies 1997). The classification into GH families, based on amino acid sequence and structural similarities, is probably the most powerful and common one, since the amino acid sequence of an enzyme gives important structural and mechanistic information of the enzyme (Henrissat 1991; Henrissat \& Davies 1997; Coutinho \& Henrissat 1999; CAZyserver http://www.cazy.org/). However, some GH families group together different enzyme activities, which complicates the prediction of gene function based on sequence alone. The starch degrading enzymes are found in only a few of the numerous GH families, mainly in GH family 13 , but also in GH family 14 and 15 (Henrissat 1991; Coutinho \& Henrissat 1999).
Family 13 glycoside hydrolases

Enzymes from GH family 13, also called the $\alpha$-amylase family, are enzymes that hydrolyse and/or transglycosylate $\alpha$-(1-4) and/or $\alpha$-(1-6) glucosidic linkages. They have a $(\beta / \alpha)_{8}$ barrel structure with four highly conserved regions in their primary structure which contain the catalytic and the major substrate-binding sites. In addition, they have conserved aspartate, glutamate and aspartate residues in the catalytic site (Kuriki \& Imanaka 1999). Several starch acting enzymes belong to this GH family 13 , such as $\alpha$-amylase (EC 3.2.1.1), maltogenic $\alpha$-amylase (EC 3.2.1.133), pullulanase (EC 3.2.1.41) and isoamylase (EC 3.2.1.68) (Coutinho \& Henrissat 1999; http://www.cazy.org/). Recently, several subfamilies have been identified (Stam et al. 2006).

The $\alpha$-amylases of $\mathrm{GH}$ family 13 have three structural domains in common, referred to as the A, B and $\mathrm{C}$ domains. The A domain is the catalytic domain and is folded into a $(\beta / \alpha)_{8}$ barrel structure. The B domain protrudes at the third $\beta$ strand of the $\mathrm{A}$ domain and is involved in substrate or $\mathrm{Ca}^{2+}$ binding. The catalytic $(\beta / \alpha)_{8}$ barrel is succeeded by the $\mathrm{C}$ domain, of which the role is largely unknown. However, it has been suggested that the $\mathrm{C}$ domain is involved in the enzyme activity (Jespersen et al. 1991; van der Maarel et al. 2002). The catalytic domain contains the active site which is composed of a catalytic site and an array of subsites, each of which interacts with individual glucose residues of the substrate (Davies et al. 1997). The number of these subsites and the position of the catalytic site depend on the amylase. In general, differences in substrate specificity of enzymes are explained by differences in the number of subsites, a different affinity for glucose residues of each subsite, different amino acid composition and differences in the catalytic site (MacGregor 1993). Many amylases possess additional domains, such as a starch binding domain (SBD). This is almost exclusively positioned at the C-terminal part of the amylase (Janecek \& Sevcik 1999) and makes it possible for the amylase to bind and hydrolyse native granular starch (Juge et al. 2006). It functions independently of the amylase. For certain $\alpha$-amylases, e.g. a raw starch degrading $\alpha$-amylase from Cryptococcus sp., this domain seems also responsible for the thermostability of the amylase (Iefuji et al. 1996). 
$\alpha$-Amylases. These are endo-acting amylases which hydrolyse the $\alpha$-(1-4) glycosidic bonds of the starch polymers internally. Upon starch hydrolysis, they form oligosaccharides with varying chain length and different $\alpha$-limit dextrins containing $\alpha-(1-6)$ bonds. Next to the main hydrolysis action of $\alpha$-(1-4) bonds, $\alpha$-amylases show also a weak transglycosylation of $\alpha-(1-4)$ bonds (Kuriki \& Imanaki 1999).

Maltogenic $\alpha$-amylases. They hydrolyse $\alpha$-(1-4) glycosidic bonds of the starch polymers, thereby liberating $\alpha$-maltose (Outtrup \& Norman 1984; Christophersen et al. 1998). Their precise mode of action is not completely clear. Some reports suggest that a maltogenic $\alpha$-amylase from Bacillus stearothermophilus (BStA) acts as an exo-amylase (Bowles 1996; Kragh 2002). However, consistent with the characteristics of an endo- $\alpha$-amylase, this enzyme does not require a non-reducing end, it does not invert the anomeric configuration (Christophersen et al. 1998), its active site is located in an open cleft (Dauter et al. 1999) and it can greatly reduce amylose molecular weight (MW) (Christophersen et al. 1998; Leman et al. 2005). Next to the three common domains (A, B and C), BStA possesses two additional domains, $\mathrm{D}$ and $\mathrm{E}$, with the $\mathrm{E}$ domain involved in binding to native granular starch (Dauter et al. 1999).

Isoamylase and pullulanase type I. These two enzymes hydrolyse exclusively $\alpha$-(1-6) glucosidic bonds. The main difference between these enzymes is the minimal length of the side chain which can be released. Isoamylase is the main starch debranching enzyme and only hydrolyses $\alpha$-(1-6) bound glucose oligomers with a DP of a least three. The main substrate of pullulanase is pullulan, but this enzyme can also hydrolyse $\alpha$-(1-6) bound maltose in amylopectin and $\beta$-limit dextrins (Hoseney 1994; van der Maarel et al. 2002).

\section{Family 14 \& 15 glycoside hydrolases}

$\beta$-Amylases. This amylase can be found in GH family 14 and has a core $(\beta / \alpha)_{8}$-barrel catalytic domain (Coutinho \& Henrissat 1999). $\beta$-Amylases are truly exo-acting amylases. They hydrolyse $\alpha$-(1-4) glycosidic bonds in starch and starch fragments and liberate $\beta$ maltose successively from the non-reducing ends of the starch polymers. These enzymes can not pass the $\alpha$ (1-6) branching points of starch. Therefore, amylose is nearly completely hydrolysed into maltose and maltotriose, while the end point of amylopectin hydrolysis consists of maltose and $\beta$-limit dextrins (Hanes 1937).

Glucoamylases. These members of GH family 15 , are generally multidomain enzymes consisting of a catalytic domain folded as a twisted $(\alpha / \alpha)_{6}$ barrel with a central funnel-shaped active site connected to a starch binding domain. Glucoamylases are inverting exo-amylases releasing $\beta$-glucose from the non-reducing ends of starch with a single displacement mechanism. They hydrolyse especially $\alpha$-(1-4) bonds but also to limited extent $\alpha$-(1-6) glucosidic bonds (Pazur \& Ando 1960). More information can be found in the reviews by, e.g., Sauer et al. (2000) and Norouzian et al. (2006).

\section{Action pattern of amylases}

Several aspects of amylase mode of action can be distinguished. A first aspect deals with the action mechanism, i.e. the hydrolysis of the glucosidic bond on a molecular scale. All the enzymes of GH family 13 retain the anomeric configuration and work according to the double displacement mechanism (MacGregor 1993; van der Maarel et al. 2002). This is an acid-base catalysed reaction, requiring a proton donor and a nucleophile, in which a glycosyl-enzyme intermediate is formed (Withers \& Aebersold 1995). Typically, all endo-acting amylases work according to this action mechanism. In contrast, exo-acting amylases, such as $\beta$-amylases, are inverting amylases and work through the single displacement mechanism (Henrissat \& Davies 1997). The reaction proceeds through an oxocarbenium ion-like transition state (Withers \& Aebersold 1995).

Another aspect of amylase mode of action is that several models for amylase action pattern have been proposed, such as the random action and the multiple attack action.

\section{Definitions}

In general, it is believed that $\alpha$-amylases degrade the starch polymers in a random manner (Gupta et al. 2003) (Fig. 2a). This has also been referred to as a single attack or multi-chain attack action (Azhari \& Lotan 1991). This mode of action implies that all bonds are equally susceptible to hydrolysis. As a result, a rapid decrease in starch polymer MW can be observed.

Amylases with a multiple attack action cleave several glycosidic bonds successively after the first (random) hydrolytic attack before dissociating from the substrate (Fig. 2b). The multiple attack action is an intermediate between the single-chain and the multichain (or random) action. In the former, the polymer molecule is successively hydrolysed completely before dissociation of the enzyme-substrate complex (Fig. 2a), while, in the latter, only one bond is hydrolysed per effective encounter (Fig. 2c) (Robyt \& French 1967). The direction of multiple attack action is towards the non-reducing end of the substrate (Robyt \& French 1970). Robyt \& French (1967) defined the degree of multiple attack (DMA) as the number of bonds broken during the lifetime of an enzyme-substrate complex minus one (i.e. the initial random cleavage). The multiple attack action is often referred to by the more generic term "processivity" (Breyer \& Matthews 2001). Many enzymes that synthesise, modify or degrade polymers, such as granule bound starch synthase I (Denyer et al. 1999), DNA polymerase (Kelman et al. 1998), and endopolygalacturonase I from Aspergillus niger (van Pouderoyen et al. 2003), act by a processive action pattern.

Some authors proposed another amylase action pattern, the so-called preferred attack action. Like in the random attack action, one bond is broken during the lifetime of an enzyme-substrate complex. However, some bonds are more susceptible to hydrolysis than oth- 
a) SINGLE CHAIN ATTACK

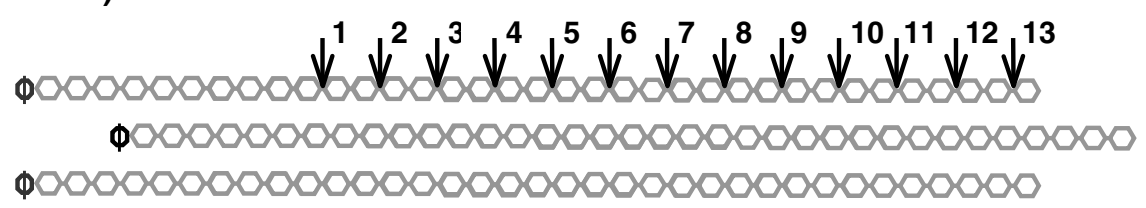

b) MULTIPLE ATTACK

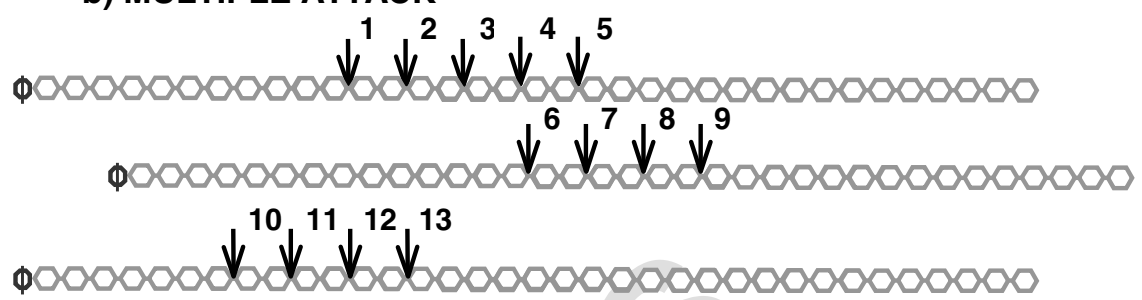

c) MULTICHAIN ATTACK

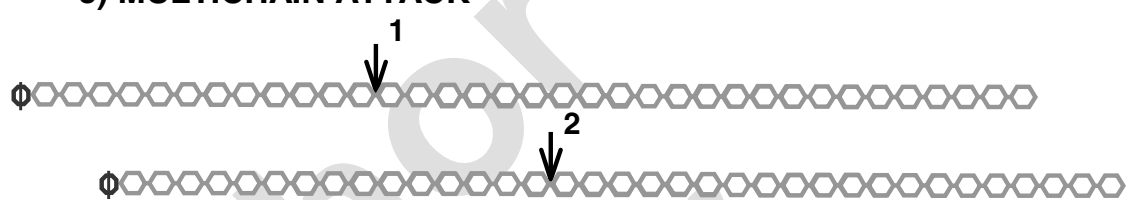

$\$ 000000 \mathbf{W}^{3} 00000000000000000000000000$

d) PREFERRED ATTACK

* * * *

o*k*

Fig. 2. Schematic representation of amylase action patterns on amylose and amylose fragments. Partially redrawn from Robyt \& French (1967). represents the glucose residues linked through $\alpha-(1-4)$ glycosidic bonds; $\varnothing$ indicates the reducing end; and arrows indicate the position of amylase attack.

ers. More in particular, bonds close to the chain ends and/or presumably also those near the branching points are less susceptible to hydrolysis. The probability for bond cleavage thus depends on the position of the bond in the chain (Thoma 1976; Banks \& Greenwood 1977).

\section{Amylases action patterns}

Reports on the multiple attack action of amylases have appeared in the literature for several decades. Many different experimental setups and substrates have been used for evaluating amylase action pattern, including amylose and series of short (labelled) oligosaccharides.

The first reports on multiple attack action. $\beta$ Amylases were the first amylolytic enzymes reported to act with a multiple attack action. Swanson (1948) suggested that $\beta$-amylase hydrolyses an amylose molecule completely to maltose before a new molecule is attacked (i.e. single chain action), since no intermediates of short chain length were detected during the action of $\beta$-amylase on amylose. Bailey \& Whelan (1957) studied the action of $\beta$-amylase on maltohexaose, malto- heptaose and a synthetic amylose with a DP of 49 . Their results indicated that the action pattern of $\beta$ amylase is intermediate between single-chain and multichain attack action (i.e. multiple attack action). Bailey $\&$ French (1957) further substantiated the multiple attack action for $\beta$-amylase by analyzing the products formed during hydrolysis of a polymer with a ${ }^{14} \mathrm{C}$ labelled non-reducing end. Results with crystalline sweet potato $\beta$-amylase indicated that the enzyme removes, on average, four units of maltose per effective enzymesubstrate encounter, at $35^{\circ} \mathrm{C}$ and $\mathrm{pH} 4.8$.

Robyt \& French (1963) studied the action pattern and specificity of a Bacillus $\alpha$-amylase. Although this enzyme was reported to originate from a B. subtilis strain, it seems that the organism was later renamed to B. amyloliquefaciens. These authors analysed the products formed during hydrolysis of amylose, potato amylopectin, the corresponding $\beta$-amylase limit dextrins, and maltooligosacharides with DP in a 2 to 12 range. For amylose and amylopectin, they found dual product specificity with formation of predominantly maltotriose 
and maltohexaose, next to smaller amounts of maltose, maltotetraose, maltopentaose and maltoheptaose. A wide range of initial products were formed when maltododecaose was used as a substrate, which demonstrates a random approach of the enzyme to the substrate. Based on their results, the Bacillus $\alpha$-amylase is an endo-acting enzyme that attacks the internal part of amylose in a random way. However, these authors suggested that after the first random attack one of the two fragments stays attached to the enzyme and further reactions proceed via an exo-multiple attack. Since no maltohexaose and maltoheptaose were formed during the hydrolysis of $\beta$-amylase limit dextrin, Robyt \& French (1963) showed that the longer oligosaccharides produced from amylopectin exclusively originated from hydrolysis of exterior amylopectin chains. The latter was later also reported by Bertoft (1989) for a B. subtilis $\alpha$-amylase (BSuA). According to Robyt \& French (1963), the amylase has a much higher probability of attacking non-reducing end external chains of amylopectin than internal chains. In their view, the action pattern of BSuA on amylopectin is of the exo-multiple attack type, yielding predominantly maltohexaose and maltoheptaose. The majority of glucose, maltose and maltotriose released from amylopectin originates from the interior chains between branching points since these parts of the chains were of a size that, after the initial cleavage, the enzyme would be able to only move over one or two glucose units.

Abdullah et al. (1966) studied $\alpha$-amylase action patterns by evaluating amylolytic action on a cyclo$\mathrm{C}_{8}$ dextrin in the presence of a large excess of $\beta$ amylase. Only endo- $\alpha$-amylases can hydrolyse the cyclic substrate. However, when the linear oligosaccharides are released, the $\beta$-amylase rapidly hydrolyses these oligosaccharides further into maltose. Upon hydrolysis of the cyclo- $\mathrm{C}_{8}$ dextrin by porcine pancreatic $\alpha$-amylase (PPA), maltotriose was formed. This indicated that PPA stays attached to the opened cyclo- $\mathrm{C}_{8}$ ring (which hence becomes unavailable for $\beta$-amylase) and performs multiple attacks before dissociating. On the other hand, for the Aspergillus oryzae $\alpha$-amylase (TAKA) only maltose was found which indicates that this amylase shows little if any multiple attack action (Abdullah et al. 1966).

The blue value method - amylose as substrate. Robyt \& French (1967) evaluated amylase action pattern using the relation between the drop in blue value [potassium iodide/iodine $\left(\mathrm{KI} / \mathrm{I}_{2}\right)$ binding, analysed at $620 \mathrm{~nm}]$ and the increase in the total reducing value during amylolysis of amylose. These authors found that PPA, TAKA and human salivary $\alpha$-amylase released a different amount of reducing sugars for a certain decrease in blue value. These differences were explained by differences in level of multiple attack action. After precipitation of the polysaccharide fraction with ethanol and measurement of its reducing value, the number of bonds broken per effective encounter can be represented by the ratio between the total level of reducing sugars formed $\left(\mathrm{RV}_{\mathrm{TS}}\right)$ to the level of reducing polysaccharides
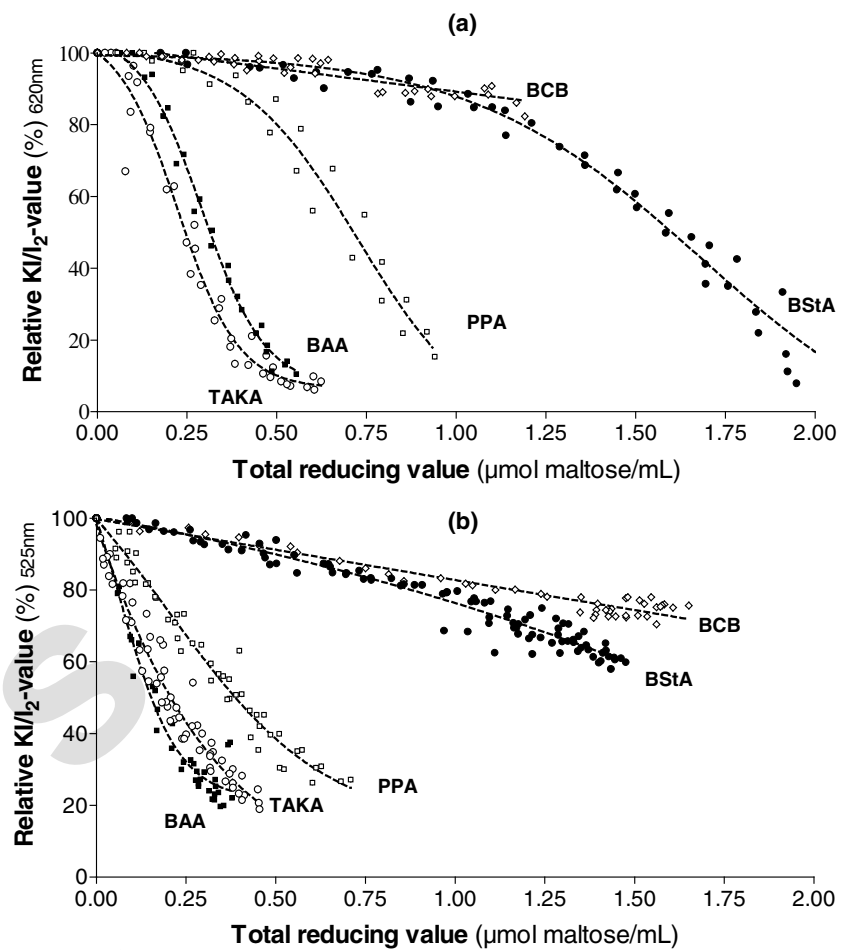

Fig. 3. Relation between the drop in relative $\mathrm{KI}^{-\mathrm{I}_{2}}$-value (as percentage of the absorbance of the aliquot at time point zero) and the increase in total reducing value during amylolysis at $35^{\circ} \mathrm{C}$ and $\mathrm{pH} 6.0$, using $0.1 \%$ potato amylose (a) or $0.1 \%$ waxy maize (b) as a substrate (Bijttebier et al. 2007a,b). (०) TAKA, Aspergillus oryzae $\alpha$-amylase; ( $\square$ BAA, Bacillus amyloliquefaciens $\alpha$-amylase; ( $\square$ ) PPA, porcine pancreatic $\alpha$-amylase; $(\bullet)$ BStA, $B$. stearothermophilus maltogenic $\alpha$ amylase; $(\diamond)$ BCB, B. cereus $\beta$ amylase. The dashed lines are the Boltzman sigmoidal lines fitted to the experimental points.

$\left(\mathrm{RV}_{\mathrm{PS}}\right)$. This model presumes that the first attack releases a polymer fragment. Therefore, the number of subsequent bonds broken (i.e. the DMA) is given by $\left(\mathrm{RV}_{\mathrm{TS}} / \mathrm{RV}_{\mathrm{PS}}\right)-1$. For PPA, TAKA and human salivary $\alpha$-amylase, DMA values of $6.0,1.9$ and 2.0 , respectively, were found (Robyt \& French 1967). Although sometimes some minor changes have been made to the setup, this method relating the decrease in iodine binding to the increase in reducing sugars is used in several other studies on amylase action pattern (Kramhøft et al. 2005; Atichokudomchai et al. 2006; Bijttebier et al. 2007a,b). They all confirmed that different amylases degrade amylose with a different level of multiple attack action, with the porcine pancreatic enzyme clearly showing the highest multiple attack action. In addition, after fitting a sigmoidal curve on the data points (Fig. 3), Bijttebier et al. (2007a,b) showed that the (calculated) reducing value corresponding to a blue value of $80 \%$ is highly correlated to the DMA value (Table 1). This way, the laborious method to calculate the DMA value (which includes a precipitation step of the polymeric amylose fragments) can be avoided. It is important to note that, in these studies, only the data obtained during the initial stages of hydrolysis (when chain end effects can be neglected) have been used to evaluate the multiple attack action (Robyt \& French 
1967; Kramhøft et al. 2005; Bijttebier et al. 2007a).

Endo-action vs. total hydrolytic actions. Since the iodine binding value of amylose is related to amylose MW (Bailey \& Whelan 1961), the blue value method of Robyt \& French (1967) can be considered as relating the first, (random) endo-attack (resulting in the decrease in MW and, thus, blue value) to the total number of hydrolysis actions (expressed by the reducing sugar levels). Other studies used other procedures to estimate this relation and thus evaluate the multiple attack action.

Klein \& Foreman (1980) compared the action patterns of human pancreatic and salivary amylase and PPA using Cibachron Blue F3GA amylose as substrate. They found that the amylases generated different proportions of reducing sugars for a certain amount of soluble dyed fragments formed. This was explained as resulting from differences in multiple attack action. Their results were in line with those obtained by Robyt \& French (1967).

Banks et al. (1970) measured the ratio of the weight-average DP to the number-average DP during the initial stages of amylose hydrolysis by BSuA and PPA. The oligosaccharides with low MW produced by multiple attack action have a significant impact on the number-average DP but only a small effect on the weight-average DP. Hence, multiple attack action would rapidly increase this ratio, while it would be constant during the early stages of a more random attack. Only for PPA a considerable level of multiple attack action was found.

Malto-oligosaccharides as substrate. Robyt \& French (1970) also studied the amylase action pattern using labelled maltooctaose. The multiple attack action of PPA was reflected in the fact that the initial rates of formation of maltose and maltotriose were higher than the rates of formation of maltohexoase and maltopentaose, respectively. Single attack would be characterised by equal initial rates of maltose and maltohexaose formation and equal initial rates of maltotriose and maltopentaose formation. Likewise, Allen \& Thoma (1978) studied the action pattern of TAKA using malto-oligosaccharides of DP 3 to 10. For the substrates maltotriose and maltotetraose, no significant level of multiple attack action was found. However, for the oligosaccharides with DP 5 to 10 a small but measurable gradual increasing extent of multiple attack action was found, since, much like Robyt \& French (1970), they observed that the smaller hydrolysis products were released to a higher extent than the larger ones. More recently, Kandra et al. (1997) studied the action pattern of PPA using model substrates [including $(4,6)$ O-benzylidene modified, nitrophenyl containing maltooligosaccharides] and confirmed the multiple attack action of this enzyme.

Multiple attack action on amylopectin? Most of the experimental evidence for the multiple attack action of amylases was obtained using the linear amylose substrate or short linear oligosaccharides. Only few reports deal with amylase action pattern on amylopectin.
Hutny \& Ugorski (1981) suggested that PPA, in contrast to $\beta$-amylase, can continue its multiple attack action along the branched substrate chains. Mazur \& Nakatani (1993) and Nakatani (1996) proposed that PPA not only acts with a multiple attack action on amylose but can also do so on the side chains of amylopectin.

Atichokudomchai et al. (2006) studied the MW distributions of the products after $\alpha$-amylolysis of a waxy maize dispersion. They found that the amylase with the lowest level of multiple attack action on amylose (or, stated differently, the highest level of random attack), as evaluated by the blue value method of Robyt \& French (1967), rapidly decreased amylopectin MW and resulted in an unimodal distribution of small branched oligosaccharides. The other two amylases with slightly higher level of multiple attack on amylose led to a bimodal distribution with both highly branched fragments and low MW oligomers, presumably resulting from the multiple attacks on amylopectin.

Bijttebier et al. (2007b) evaluated amylase action pattern with amylopectin as substrate using the relation between the drop in $\mathrm{KI} / \mathrm{I}_{2}$ binding (analysed at $525 \mathrm{~nm}$ ) and the increase in the total reducing value (Fig. 3b). Like for amylose, the different amylases released a different amount of reducing sugars for a certain decrease in iodine binding value, but the differences between PPA and the other endo- $\alpha$-amylases were much smaller than for amylose. These differences were primarily explained by differences in the level of multiple attack action. However, it should be taken into account that the procedure based on the iodine binding of amylopectin is much less sensitive than the amylose blue value method.

Multiple attack or preferred attack? Bird \& Hopkins (1954) studied the action of $\alpha$-amylases from $B$. subtilis, malt and saliva on amylose by analysing the products generated during amylolysis. They concluded that the hydrolysis of amylose was random with the exception of some linkages, mostly in the vicinity of the chain ends. At the later stages of hydrolysis, the number of chain ends becomes higher and the nonsusceptibility of some bonds becomes the controlling factor (i.e. preferred attack action). For the malt and bacterial $\alpha$-amylase the second to the fifth linkage from the non-reducing end and the first or first two bonds at the reducing end of an amylose molecule are attacked with great difficulty. For the salivary amylase, hydrolysis of the end-linkages and the second and third linkage from the non-reducing end are exceptions to the random attack action (Bird \& Hopkins 1954).

Thoma (1976) aimed to elucidate the action pattern of $\alpha$-amylases based on mathematical models. Experimental data obtained by studying the effects of $\mathrm{pH}$, inhibitors and substrate chain length on the action pattern of amylases, were used to evaluate the mathematical models of random, preferred attack and multiple attack action. For PPA, experimental data were consistent with the model for multiple attack action. However, some of the experimental data were also consistent 
with a preferred attack action. In contrast, B. amyloliquefaciens $\alpha$-amylase (BAA) hydrolyses oligosaccharides exclusively by preferred attack, as it tends to cleave the three bonds nearest to the reducing end. However, for longer chain lengths, BAA predominantly showed a random attack action in the interior of the molecules. Thoma (1976) pointed out that experiments designed to determine the action pattern of an amylase must take into account the effect of the size of the substrate. A single enzyme may work by a combination of mechanisms and the mechanism that dominates will depend on the substrate chain length. Indeed, when PPA acts on a very long substrate, it has a random or multiple attack action. However, when chain ends become numerous, the preferred attack action becomes important. At the end of hydrolysis, when the oligosaccharides are dominant, the preferred attack action will even be the only action pattern possible (Thoma 1976). Likewise, Allen \& Thoma (1978) found for TAKA a gradual increase of multiple attack action with increasing substrate length.

Like Thoma (1976), Banks \& Greenwood (1977) developed mathematical models for multiple attack and preferred attack action patterns. Both models were applied to the experimental results obtained by Robyt \& French (1967) and Banks et al. (1970) in order to determine whether the multiple attack action is important in the action pattern of amylases. The multiple attack action could only be confirmed for PPA, while the other $\alpha$-amylases were mainly random acting enzymes.

The above clearly shows that the preferred attack action of amylases is a valuable concept to explain the non-random distribution of oligosaccharides in the amylase digest of starch polymers. However, we believe this action pattern should be considered in a different way than the single or multiple attack actions. In our view, the preferred attack action is one way to describe amylase substrate specificity and oligosaccharide cleavage patterns, as determined by active site architecture. Indeed, the number of subsites and the position of the catalytic residues in the active site, which depend on the enzyme (MacGregor 1993), determine productive substrate binding and the resulting malto-oligosaccharides product distribution. In the ideal arrangement, all substrate binding subsites in the active site should be filled by a glucose residue. However, particularly in the case of short oligosaccharides and near chain ends, not all possible interactions between substrate and active site (particularly the substrate binding subsites) result in the ideal arrangement and/or productive binding. This is in line with the findings that the preferred attack action is usually linked to oligosaccharide degradation or the later stages of polysaccharide hydrolysis, when chain end effects become more pronounced. In contrast, during the early stages of hydrolysis, when the substrate is of sufficiently high DP, hydrolysis is more or less random (Thoma 1976; Banks \& Greenwood 1977) and amylase action pattern should be discussed in terms of single or multiple attack action. Since amylopectin is made up by interlinked linear short chains, it is conceiv- able that chain end effects will be important in amylopectin hydrolysis as well.

In short, from this literature overview, it can clearly be seen that the multiple attack action is generally an accepted concept to explain the differences in action pattern of amylases (Svensson et al. 2002; Kramhøft et al. 2005). However, most of the endo- $\alpha$ amylases have a low to very low level of multiple attack action. The pancreatic $\alpha$-amylase remains one of the few enzymes known with a considerable level of multiple attack action.

Influence of $p H$ and temperature on the action pattern Few reports deal with the influence of $\mathrm{pH}$ and temperature on the action pattern of amylases.

Bailey \& Whelan (1957) showed that with increasing $\mathrm{pH}$, the action pattern of a $\beta$-amylase becomes more random. Likewise, Robyt \& French (1967, 1970) reported that upon increasing the $\mathrm{pH}$ from the optimum $\mathrm{pH} 6.9$ to 10.5 the action pattern of PPA changes from a multiple attack to a single attack action. However, decreasing the $\mathrm{pH}$ to 4.5 conserved its multiple attack action (Robyt \& French 1967, 1970). According to Robyt \& French (1967), one or more of the catalytic groups can probably not function more than once during the lifetime of an enzyme-substrate complex due to an unfavourable ionisation state at high $\mathrm{pH}$.

Ishikawa et al. (1991) studied the hydrolysis of ${ }^{14} \mathrm{C}$ labelled malto-oligosaccharides by PPA and found a minor effect of $\mathrm{pH}$ on the formation of maltotriose and maltotetraose and no effect of $\mathrm{pH}$ on the formation of higher oligosaccharides (of DP 5 and higher). Marchal et al. (1999) investigated the oligosaccharide composition obtained after hydrolysis of potato starch by $B$. licheniformis $\alpha$-amylase (BLA), but noted no effect of $\mathrm{pH}$. However, the $\mathrm{pH}$ values in the latter study only varied between $\mathrm{pH} 5.1$ and 7.6 and this range may be too small to greatly affect amylase action pattern.

Bailey \& Whelan (1957) also found that, at optimum $\mathrm{pH}$, the level of single attack action of $\beta$-amylase decreased with increasing temperature between 0 and $20^{\circ} \mathrm{C}$, was more or less constant between 20 and $60^{\circ} \mathrm{C}$ and increased when temperature was raised between 60 and $75^{\circ} \mathrm{C}$. Mazur \& Nakatani (1993) found that the product distribution during amylolysis by PPA depends on the temperature and that the level of multiple attack action increases with increasing temperature. For BLA, Kramhøft \& Svensson (1998) did not notice any effect on DMA when the temperature was raised from 37 to $80^{\circ} \mathrm{C}$. Bijttebier et al. (2007a) showed that the level of multiple attack (based on calculated $R_{80}$ values derived from plots of blue value data against total reducing sugars) of several endo- $\alpha$-amylases increased with temperature to a degree depending on the amylase (Table 1). In contrast, these authors found for BStA that its level of multiple attack action decreased when temperature was raised. This indicates that this maltogenic enzyme has a more pronounced endo-action at higher temperatures (Table 1). Furthermore, Marchal et al. (1999) reported that, for the same level of bonds 
Table 1. Level of multiple attack action of amylases, represented by degree of multiple attack (DMA) and $\mathrm{RV}_{80}$ values. $^{a}$

\begin{tabular}{|c|c|c|c|c|}
\hline \multirow{2}{*}{ Enzyme } & \multirow{2}{*}{$\frac{\text { DMA }}{35^{\circ} \mathrm{C}}$} & \multicolumn{3}{|c|}{$\mathrm{RV}_{80}$} \\
\hline & & $35^{\circ} \mathrm{C}$ & $50^{\circ} \mathrm{C}$ & $70^{\circ} \mathrm{C}$ \\
\hline TAKA & $2.5 \pm 0.2 \mathrm{a}$ & $0.14 \pm 0.02 \mathrm{a}$ & $0.25 \pm 0.04 \mathrm{c}$ & n.d. \\
\hline BAA & $3.9 \pm 0.4 \mathrm{~b}$ & $0.20 \pm 0.02 \mathrm{~b}$ & $0.17 \pm 0.01 \mathrm{~b}$ & $0.23 \pm 0.05 \mathrm{bc}$ \\
\hline PPA & $8.0 \pm 0.7 \mathrm{c}$ & $0.51 \pm 0.06 \mathrm{~d}$ & $0.46 \pm 0.02 \mathrm{~d}$ & n.d. \\
\hline BStA & $12.1 \pm 2.1 \mathrm{~d}$ & $1.18 \pm 0.03 \mathrm{e}$ & $0.94 \pm 0.03 \mathrm{f}$ & $0.73 \pm 0.02 \mathrm{~g}$ \\
\hline
\end{tabular}

$a$ The data are from Bijttebier et al. (2007a). $\mathrm{RV}_{80}$ (i.e. the reducing value for a $\mathrm{KI}_{-} \mathrm{I}_{2}$-value of $80 \%$ ) is a parameter calculated from the Boltzmann sigmoidal regression equation, which was fitted on the plot of the amylose $\mathrm{KI}-\mathrm{I}_{2}$ binding value data against the total reducing value during amylolysis, as described by Bijttebier et al. (2007a). Reaction conditions: pH 6.0, 0.1\% potato amylose as a substrate. Different letters indicate significantly different DMA or $\mathrm{RV}_{80}$ values as determined by a $t$-test with $P<0.05$. Values are means with corresponding standard deviations of at least three individual experiments. TAKA, Aspergillus oryzae $\alpha$-amylase; BAA, Bacillus amyloliquefaciens $\alpha$-amylase; PPA, porcine pancreatic $\alpha$-amylase, BStA, B. stearothermophilus maltogenic $\alpha$-amylase. n.d., not detectable.

hydrolysed, the oligosaccharide composition, obtained after hydrolysis of starch by BLA, BAA and an $\alpha$ amylase from B. stearothermophilus, depends on the hydrolysis temperature. The product specificity decreased with temperature and a more uniform distribution of the hydrolysis products was found.

\section{Mechanism of multiple attack}

The molecular basis of the multiple attack action mechanism is not well understood. Several molecular mechanisms were proposed to explain the multiple attack action of amylases (Robyt \& French 1970).

A first hypothesis is related to the cage effect of the solvent. This means that the solvent does not allow the starch polymer fragments to quickly diffuse away after hydrolysis. Thus, the dissociation of the two fragments from the enzyme is rapidly followed by a reassociation of one of the two fragments to the enzyme. In this case, it would be expected that amylases, such as PPA and human salivary $\alpha$-amylase, with similar molecular size and acting on the same substrate and under identical conditions, show a similar level of multiple attack. However, the action patterns of PPA and human salivary $\alpha$-amylase are completely different (Robyt \& French 1967). Moreover, this mechanism cannot explain the high DMA and observed $\mathrm{pH}$ dependence of the multiple attack action of PPA (Robyt \& French 1967; Mazur \& Nakatani 1993). Hence, differences in amylase action patterns are probably due to intrinsic differences in enzyme properties.

A second hypothesis is referred to as the sliding mechanism, in which an amylase can move along the substrate without dissociation (Robyt \& French 1970; Mazur \& Nakatani 1993). In this view, binding between substrate and enzyme should be rather weak and non-specific and is assumed to occur through hydrogen bonds, van der Waals contacts and stacking interactions with aromatic side chains (Mazur \& Nakatani 1993; Breyer \& Mathews 2001; Kramhøft et al. 2005; Ishikawa et al. 2007). According to Mazur \& Nakatani (1993), the sliding mechanism is in line with the dependence of the action pattern on temperature and substrate chain length. With increasing chain length, the hydrophobic binding area increases, leading to an increased probability of sliding, which, in its turn, is probably reflected in the observed increase of multiple attack action. Likewise, several studies reported an increased multiple attack action when temperature was raised. In this context, the free energy barrier of sliding is an enthalpic event and the hydrophobic stacking has a great entropic contribution to the free energy of dissociation. Therefore, increasing temperature will increase sliding velocity, while the rate of dissociation only increases to a limited extent or may even decrease (Mazur \& Nakatani 1993).

Several authors have commented on the structural requirements, particularly related to the enzymesubstrate interaction, of the amylases for multiple attack action. This has already been discussed to some extent by Bijttebier et al. (2007a) and is here presented more elaborately.

According to Breyer \& Matthews (2001), processivity of enzymes is related to the degree of enclosure of the substrate by the enzyme. The cleft-shaped binding sites, found in endo- $\alpha$-amylases, and the pocket-shaped active site of exo-amylases, both allow a partial enclosure of the substrates. However, not all amylases have a high level of multiple attack action, demonstrating that the general shape of the active site is not the only prerequisite for multiple attack action. In this context, Breyer \& Matthews (2001) also proposed that processivity can be achieved by the enzyme having a large interaction surface with the substrate.

A first aspect of the interaction between enzyme and substrate can be related to the active site, and the array of glucose binding subsites within. Kramhøft \& Svensson (1998) proposed a possible relation between the number of subsites and DMA. They observed a high DMA for PPA having five subsites and a low DMA for amylases with nine to ten subsites. In this view, and, somewhat in contrast to the suggestion by Breyer \& Matthews (2001), amylases with a shorter substrate binding cleft (lower number of subsites) have a higher DMA. Similar observations have been made by Bijttebier et al. (2007a). Furthermore, Bozonnet et al. (2003), Kramhøft et al. (2005) and Ishikawa et al. (2007) clearly showed the importance of the subsites in determining DMA. Using barley $\alpha$-amylase AMY-1 mutants, Bozon- 
net et al. (2003) and Kramhøft et al. (2005) observed that structural changes in the substrate binding cleft altered the DMA of this enzyme. More in particular, a single mutation at subsite -6 (i.e. the non-reducing end of substrate binding site) leads to a looser substrate binding at this subsite and increases DMA (Kramhøft et al. 2005). However, other mutations can counteract this increase, or can even drastically lower the DMA. These authors suggested that changes in the substrate binding cleft can result in a less efficient repositioning of substrate without dissociation after hydrolysis (substrate sliding). This is possibly due to difficulty in accommodating the stable left-hand helical substrate conformation. Ishikawa et al. (2007) identified some amino acid residues, which are critical for the multiple attack action of soybean $\beta$-amylase. Their data showed that the carboxyl residue of Asp53 stabilises the glucose residue of the substrate at binding subsite -2 , which is necessary for the multiple attack action. Furthermore, Trp55, which is located in the vicinity of the nonreducing end of substrate binding site, was also found to be critical for the multiple attack action of the soybean $\beta$-amylase. Indeed, mutation of this residue into an arginine residue completely eliminated the multiple attack action. This mutation changed the orientation of Asp53, as observed in the crystal structure of the mutant enzyme, thus reducing the binding affinity of subsite -2 . These data suggest that substrate binding in the active site binding subsites is crucial for multiple attack action. The interactions should be weak enough to allow sliding of the substrate in the active site, but strong enough to stabilize the productive enzyme/substrate complex after sliding (Ishikawa et al. 2007).

A second aspect of the interaction between enzyme and substrate is related to the presence of carbohydrate binding regions outside the active site. In this respect, the fusion of AMY-1 with an SBD increases DMA (Kramhøft et al. 2005; Juge et al. 2006). Likewise, the high DMA-values for the maltogenic BStA and PPA (Bijttebier et al., 2007a) are in agreement with the importance of such starch interaction sites in determining DMA. Indeed, putative starch interaction sites outside the active site have been identified for BStA (i.e. a starch binding domain E) (Dauter et al. 1999) and PPA (i.e. two independent carbohydrate recognition sites) (Qian et al. 1995). In the latter case, they have been suggested to be involved in the multiple attack action. However, some enzymes with a low level of multiple attack action possess (putative) starch or maltose interaction sites as well. This would indicate that their low DMA-values are primarily determined by the large number of subsites or the architecture of their active site (Bijttebier et al. 2007a). Based on these considerations, Bijttebier et al. (2007a) concluded that the multiple attack action of an amylase is probably related to the size of the interaction surface between enzyme and substrate (as positively affected by the presence of substrate binding domains or carbohydrate recognition sites outside the active site) and to the degree to which the active site architecture and properties (as influenced by the number of subsites or the specific amino acids in those subsites) allow the reorganization (sliding) of the substrate with its own specific helical conformation.

\section{Acknowledgements}

This research was conducted in the framework of research project G.0427.07, financed by the Fund for Scientific Research - Flanders (FWO) (Brussels, Belgium), and is part of the Methusalem programme "Food for the Future" at the K.U. Leuven.

\section{References}

Abdullah M., French D. \& Robyt J.F. 1966. Multiple attack by $\alpha$-amylases. Arch. Biochem. Biophys. 114: 595-598.

Allen J.D. \& Thoma J.A. 1978. Repetitive attack by Aspergillus oryzae $\alpha$-amylase. Carbohyd. Res. 61: 377-385.

Atichokudomchai N., Jane J.L. \& Hazlewood G. 2006. Reaction pattern of a novel thermostable $\alpha$-amylase. Carbohydr. Polym. 64: 582-588.

Azhari R. \& Lotan N. 1991. Enzymic hydrolysis of biopolymers via single-scission attack pathways: a unified kinetic model. J. Mater. Sci. Mater. Med. 2: 9-18.

Bailey J.M. \& French D. 1957. The significance of multiple reactions in enzyme-polymer systems. J. Biol. Chem. 226: 1-14.

Bailey J.M. \& Whelan W.J. 1957. Mechanism of carbohydrase action 3. Action pattern of $\beta$-amylase. Biochem. J. 67: 540547.

Bailey J.M. \& Whelan W.J. 1961. Physical properties of starch I. Relationship between iodine stain and chain length. J. Biol. Chem. 236: 969-972.

Banks W. \& Greenwood C.T. 1977. Mathematical models for action of $\alpha$-amylase on amylose. Carbohydr. Res. 57: 301315.

Banks W., Greenwood C.T. \& Khan K.M. 1970. Studies on starch-degrading enzymes Part XII. The initial stages of the action on amylose of the $\alpha$-amylases from B. subtilis, human saliva, malted rye, and porcine pancreas. Carbohydr. Res. 12: 79-87.

Banks W., Greenwood C.T. \& Khan K.M. 1971. Physicochemical studies on starches 56 . The interaction of linear amylose oligomers with iodine. Carbohydr. Res. 17: 25-33.

Bertoft E. 1989. Investigation of the fine-structure of amylopectin using $\alpha$-amylase and $\beta$-amylase. Carbohydr. Res. 189: 195207.

Bijttebier A., Goesaert H. \& Delcour J.A. 2007a. Temperature impacts the multiple attack action of amylases. Biomacromolecules8: 765-772.

Bijttebier A., Goesaert H. \& Delcour J.A. 2007b. Action pattern of different amylases on amylose and amylopectin, p. 52. In: Janecek S. (ed.), $3^{\text {rd }}$ Symposium on the Alpha-Amylase Family, Programme and Abstracts, Sep 23-27, 2007, Smolenice Castle, Slovakia, Asco Arts \& Science, Bratislava.

Bird R. \& Hopkins R.H. 1954. The action of some $\alpha$-amylases on amylose. Biochem. J. 56: 86-99.

Bowles L.K. 1996. Amylolytic enzymes, pp. 105-129. In: Hebeda E.H. \& Zobel H.F. (eds), Baked Goods Freshness: Technology, Evaluation and Inhibition of Staling, Marcel Dekker Inc., New York.

Bozonnet S., Kim T.J., Bonsager B.C., Kramhøft B., Nielsen P.K., Bak-Jensen K.S. \& Svensson B. 2003. Engineering of barley $\alpha$-amylase. Biocatal. Biotransform. 21: 209-214.

Breyer W.A. \& Matthews B.W. 2001. A structural basis for processivity. Protein Sci.10: 1699-1711.

Buléon A., Colonna P., Planchot V. \& Ball S. 1998. Starch granules: structure and biosynthesis. Int. J. Biol. Macromol. 23: $85-112$. 
Christophersen C., Otzen D.E., Norman B.E., Christensen S. \& Schäfer T. 1998. Enzymatic characterisation of Novamyl, a thermostable $\alpha$-amylase. Starch/Stärke 50: 39-45.

Coutinho P.M. \& Henrissat B. 1999. Carbohydrate-active enzymes: an integrated database approach, pp 3-12. In: Gilbert H.J., Davies G., Henrissat B. \& Svensson B. (eds), Recent Advances in Carbohydrate Bioengineering, The Royal Society of Chemistry, Cambridge; URL: http://www.cazy.org/.

Dauter Z., Dauter M., Brzozowski A.M., Christensen S., Borchert T.V., Beier L., Wilson K.S. \& Davies G.J. 1999. X-ray structure of Novamyl, the five-domain "Maltogenic" $\alpha$-amylase from Bacillus stearothermophilus: maltose and acarbose complexes at $1.7 \AA$ resolution. Biochemistry 38: 8385-8392.

Davies G.J., Wilson K.S. \& Henrissat B. 1997. Nomenclature for sugar-binding subsites in glycosyl hydrolases. Biochem. J. 321: $557-559$.

Denyer K., Waite D., Motawia S., Moller B.L. \& Smith A.M. 1999. Granule-bound starch synthase I in isolated starch granules elongates malto-oligosaccharides processively. Biochem. J. 340: 183-191.

Gupta R., Gigras P., Mohapatra H., Goswami V.K. \& Chauhan B. 2003. Microbial $\alpha$-amylases: a biotechnological perspective. Process Biochem. 38: 1599-1616.

Hanes C.S. 1937. The action of amylases in relation to the structure of starch and its metabolism in the plant. Parts IV-VII. New Phytol. 36: 189-282.

Henrissat B. 1991. A classification of glycosyl hydrolases based on amino acid sequence similarities. Biochem. J. 280: 309-316.

Henrissat B. \& Davies G. 1997. Structural and sequence-based classification of glycoside hydrolases. Curr. Opin. Struct. Biol. 7: $637-644$.

Hizukuri S. 1986. Polymodal distribution of the chain lengths of amylopectin and its significance. Carbohydr. Res. 147: 342347.

Hizukuri S. 1996. Starch: analytical aspects, pp. 347-429. In: Eliasson A.C. (ed.), Carbohydrates in Food, Marcel Dekker, New York.

Hizukuri S., Takeda Y. \& Yasuda M. 1981. Multi-branched nature of amylose and the action of debranching enzymes. Carbohydr. Res. 94: 205-213.

Hoseney R.C. 1994. Starch, pp. 29-64. In: Principles of Cereal Science and Technology, American Association of Cereal Chemists, St. Paul, MN.

Hutny J. \& Ugorski M. 1981. Kinetics of hog pancreas $\alpha$-amylase, development of the multiple attack model. Arch. Biochem. Biophys. 206: 29-42.

Iefuji H., Chino M., Kato M. \& Iimura Y. 1996. Raw-starchdigesting and thermostable $\alpha$-amylase from the yeast Cryptococcus sp. S-2: purification, characterization, cloning and sequencing. Biochem. J. 318: 989-996.

Ishikawa K., Matsui I., Honda K., Kobayashi S. \& Nakatani H. 1991. The $\mathrm{pH}$ dependence of the action pattern in porcine pancreatic $\alpha$-amylase catalyzed reaction for maltooligosaccharide substrates. Arch. Biochem. Biophys. 289: 124-129.

Ishikawa K., Nakatani H., Katsuya Y. \& Fukazawa C. 2007. Kinetic and structural analysis of enzyme sliding on a substrate: multiple attack in $\beta$-amylase. Biochemistry 46: 792-798.

Jane J., Chen Y.Y., Lee L.F., McPherson A.E., Wong K.S., Radosavljevic M. \& Kasemsuwan T. 1999. Effects of amylopectin branch chain length and amylose content on the gelatinization and pasting properties of starch. Cereal Chem. 76: 629-637.

Janecek S. \& Sevcik J. 1999. The evolution of starch-binding domain. FEBS Lett. 456: 119-125.

Jespersen H.M., MacGregor E.A., Sierks M.R. \& Svensson B. 1991. Comparison of the domain-level organization of starch hydrolases and related enzymes. Biochem. J. 280: 51-55.

Juge N., Nohr J., Le Gal-Coeffet M.F., Kramhøft B., Furniss C.S.M., Planchot V., Archer D.B., Williamson G. \& Svensson B. 2006. The activity of barley $\alpha$-amylase on starch granules is enhanced by fusion of a starch binding domain from $A s$ pergillus niger glucoamylase. Biochim. Biophys. Acta 1764: 275-284.

Kandra L., Gyemant G., Farkas E. \& Liptak A. 1997. Action pattern of porcine pancreatic $\alpha$-amylase on three different series of $\beta$-maltooligosaccharide glycosides. Carbohydr. Res. 298: $237-242$.

Kelman Z., Hurwitz J. \& O'Donnell M. 1998. Processivity of DNA polymerases: two mechanisms, one goal. Structure 6: 121125.

Klein B. \& Foreman J.A. 1980. Amylolysis of a chromogenic substrate, Cibachron Blue F3GA-amylose: kinetics and mechanism. Clin. Chem. 26: 250-253.

Kragh K.M. 2002. Amylases in baking, pp. 221-226. In: Courtin C.M., Veraverbeke W.S. \& Delcour J.A. (eds), Recent Advances in Enzymes in Grain Processing, Laboratory of Food Chemistry, K.U. Leuven, Leuven.

Kramhøft B., Bak-Jensen K.S., Mori H., Juge N., Nohr J. \& Svensson B. 2005. Involvement of individual subsites and secondary substrate binding sites in multiple attack on amylose by barley $\alpha$-amylase. Biochemistry 44: 1824-1832.

Kramhøft B. \& Svensson B. 1998. Effect of temperature and $\mathrm{Ca}^{2+}$ on the degree of multiple attack exhibited by mesophilic and thermophilic $\alpha$-amylases pp. 343-347. In: Ballasteros A., Plou F.J., Iborra J.L. \& Halling P.J. (eds), Progress in Biotechnology 15, Stability and Stabilization of Biocatalysts, Elsevier, Amsterdam.

Kuriki T. \& Imanaka T. 1999. The concept of the $\alpha$-amylase family: structural similarity and common catalytic mechanism. J. Biosci. Bioeng. 87: 557-565.

Leman P., Goesaert H., Vandeputte G.E., Lagrain B. \& Delcour J.A. 2005. Maltogenic amylase had a non-typical impact on the molecular and rheological properties of starch. Carbohydr. Polym. 62: 205-213.

MacGregor E.A. 1993. Relationships between structure and activity in the $\alpha$-amylase family of starch-metabolizing enzymes. Starch/Stärke 45: 232-237.

Manners D.J. 1979. The enzymatic degradation of starches, pp. 5-91. In: Blanshard J.M.V. \& Mitchell J.R. (eds), Polysaccharides in Food, Butterworths, London.

Marchal L.M., van de Laar A.M.J., Goetheer E., Schimmelpennink E.B., Bergsma J., Beeftink H.H. \& Tramper J. 1999. Effect of temperature on the saccharide composition obtained after $\alpha$-amylolysis of starch. Biotechnol. Bioeng. 63: 344-355.

Mazur A.K. \& Nakatani H. 1993. Multiple attack mechanism in the porcine pancreatic $\alpha$-amylase hydrolysis of amylose and amylopectin. Arch. Biochem. Biophys. 306: 29-38.

Mukerjea R., Slocum G., Mukerjea R. \& Robyt J.F. 2006. Significant differences in the activities of $\alpha$-amylases in the absence and presence of polyethylene glycol assayed on eight starches solubilized by two methods. Carbohydr. Res. 341: 2049-2054.

Nakatani H. 1996. Monte Carlo simulation of multiple attack mechanism of $\alpha$-amylase. Biopolymers 39: 665-669.

Norouzian D., Akbarzadeh A., Scharer J.M. \& Young M.M. 2006. Fungal glucoamylases. Biotechnol. Adv. 24: 80-85.

Outtrup H. \& Norman B.E. 1984. Properties and application of a thermostable maltogenic amylase produced by a strain of Bacillus modified by recombinant-DNA techniques. Starch/Stärke 36: 405-411.

Pazur J.H. \& Ando T. 1960. The hydrolysis of glucosyl oligosaccharides with $\alpha$-D- $(1,4)$ and $\alpha$-D- $(1,6)$ bonds by fungal amyloglucosidase. J. Biol. Chem. 235: 297-302.

Qian M.X., Haser R. \& Payan F. 1995. Carbohydrate binding sites in a pancreatic $\alpha$-amylase-substrate complex, derived from X-ray structure analysis at $2.1 \AA$ resolution. Protein Sci. 4: $747-755$.

Robyt J.F. \& French D. 1963. Action pattern and specificity of an amylase from Bacillus subtilis. Arch. Biochem. Biophys. 100: $451-467$.

Robyt J.F. \& French D. 1967. Multiple attack hypothesis of $\alpha$ amylase action: action of porcine pancreatic, human salivary, and Aspergillus oryzae $\alpha$-amylases. Arch. Biochem. Biophys. 122: $8-16$.

Robyt J.F. \& French D. 1970. Multiple attack and polarity of action of porcine pancreatic $\alpha$-amylase. Arch. Biochem. Biophys. 138: $662-670$.

Sauer J., Sigurskjold B.W., Christensen U., Frandsen T.P., Mirgorodskaya E., Harrison M., Roepstorff P. \& Svensson B. 2000. Glucoamylase: structure/function relationships, and protein engineering. Biochim. Biophys. Acta 1543: 275-293. 
Singh N., Singh J., Kaur L., Sodhi N.S. \& Gill B.S. 2003. Morphological, thermal and rheological properties of starches from different botanical sources. Food Chem. 81: 219-231.

Song Y. \& Jane J. 2000. Characterization of barley starches of waxy, normal and high amylose varieties. Carbohydr. Polym. 41: $365-377$.

Stam M.R., Danchin E.G.J., Rancurel C., Coutinho P.M. \& Henrissat B. 2006. Dividing the large glycoside hydrolase family 13 into subfamilies: towards improved functional annotations of $\alpha$-amylase-related proteins. Protein Eng. Des. Sel. 19: 555562 .

Svensson B., Jensen M.T., Mori H., Bak-Jensen K.S., Bonsager B., Nielsen P.K., Kramhøft B., Praetorius-Ibba M., Nohr J., Juge N., Greffe L., Williamson G. \& Driguez H. 2002. Fascinating facets of function and structure of amylolytic enzymes of glycoside hydrolase family 13. Biologia 57 (Suppl. 11): $5-19$.

Swanson M.A. 1948. Studies on the structure of polysaccharides II. Degradation of polysaccharides by enzymes. J. Biol. Chem. 172: $805-814$.
Thoma J.A. 1976. Models for depolymerizing enzymes - criteria for discrimination of models. Carbohydr. Res. 48: 85-103. van der Maarel M.J.E.C., van der Veen B., Uitdehaag J.C.M., Leemhuis H. \& Dijkhuizen L. 2002. Properties and applications of starch-converting enzymes of the $\alpha$-amylase family. J. Biotechnol.94: 137-155.

van Pouderoyen G., Snijder H.J., Benen J.A.E. \& Dijkstra B.W. 2003. Structural insights into the processivity of endopolygalacturonase I from Aspergillus niger. FEBS Lett. 554: 462466.

Withers S.G. \& Aebersold R. 1995. Approaches to labeling and identification of active site residues in glycosidases. Protein Sci. 4: 361-372.

Zobel H.F. 1988. Molecules to granules: a comprehensive review. Starch/Stärke 40: 1-7.

Received June 30, 2008 Accepted August 14, 2008 